\title{
Numerical Simulation on the Radar Cross Section of Variable-Sweep Wing Aircraft
}

\author{
Shichun Chen'1, Kuizhi Yue ${ }^{1,2}$, Bing Hu¹, Rui Guo
}

\begin{abstract}
The influence of variable-sweep wing on the aircraft's radar cross section (RCS) characteristics has been studied to reduce the aircraft's RCS as well as its detection probability by the hostile radar. With the help of CATIA, a 3-D digital model of the variable-sweep wing aircraft is built to generate a series of digital grids. Using MATLAB, a numerical simulation on the RCS of variable-sweep wing aircraft is conducted based on physical optics (PO) method and equivalent currents method (ECM). The results of mathematical statistics and comparative analysis show that: (i) the RCS peak value in the head direction of the aircraft decreases non-linearly with the sweep angle of the wing's leading edge; (ii) the azimuth angle corresponding to one of the peak values of the aircraft's RCS is equal to the leading edge's sweep angle; (iii) when the leading edge's sweep angle is $33^{\circ}$, the arithmetic average value of the RCS values in the head direction of the aircraft is $0.644 \%$ of the average value when the sweep angle is $0^{\circ}$; (iv) the larger the sweep angle is, the lower the probability that the aircraft is detected.
\end{abstract}

KEYWORDS: Aircraft conceptual design, Radar cross section, Variable-sweep wing, Stealth performance, Numerical simulation.

\section{INTRODUCTION}

Stealth aircraft or low-observability aircraft is a very important developing direction for modern combat aircraft. In view of this trend, the main military powers all over the world are conducting studies on the stealth aircraft's designing, manufacturing, arming and application. Typical stealth aircraft developed by the U.S. include F-22, B-2, F-35, X-45 and X-47 (Nangia and Palmer, 2005; Vogel, 2005). These aircraft have become or will become the backbone elements of the U.S. Air Force and U.S. Navy. Russia is also developing its Su-47, MG-1.44, T-50, and other stealth combat aircraft, which are likely to become the future main force of the Russia Air Force. Chinas aviation industry has also taken part in this competition and has achieved considerable success in developing its stealth combat aircraft. Variable-sweep wing aircraft have a glorious history. Classical aircraft such as the U.S. F-14, F-111, have participated in many wars. They are once the real backbone elements of the U.S. Navy and Air Force. In Russia, MG-23, $\mathrm{Su}-24, \mathrm{Tu}-160$, and other aircraft are still performing a variety of combat missions. This paper conducts feasibility studies on the stealth design of the variable-sweep wing aircraft. The RCS features are explored to provide reference and theoretical support for the aircraft's conceptual design and stealth optimization and thus provide technical reserves for the development of new stealth aircraft.

Researchers have performed lots of work on designing stealth aircraft for many years and have achieved quite many results. The different shapes of the stealth aircraft, including the aircraft's head, fuselage, wings, inlets and other strong scattering parts of electromagnetic waves, have been studied (Wood and Bauer, 2001). Stealth materials are

\footnotetext{
三 1.Beijing University of Aeronautics and Astronautics - School of Aeronautic Science and Engineering - Beijing 100191 - China. 2.Naval Aeronautical and Astronautical University - Department of Airborne Vehicle Engineering - Yantai 264001 - China.

Author for correspondence: Yue Kuizhi | Beijing University of Aeronautics and Astronautics - School of Aeronautic Science and Engineering | Beijing 100191 - China Email: yuekuizhi_2000@sohu.com

Received: 09/21/2014 | Accepted: 04/13/2015
} 
analyzed and the key technologies of radar stealth, infrared stealth, visible and acoustic stealth are explored as well. Moreover, multidisciplinary optimization algorithms on pneumaticity, stealth and structure are investigated. Bai and Liu (2007) have studied the parametric modeling approach for fuselage sections. Taking into account the aircraft's stealth performance, their approach generates the body shape by adjusting the body parameters. Tom and Alfred (2010) have studied the relationship between the aircraft's configuration and its stealth performance when the resistance is the lowest. In Bao and Wang (2012), the characteristic parameters of flying wing's stealth performance and aerodynamic performance are investigated, and a wind tunnel test of the flying wing is conducted. Ji et al. (2009) have studied the impact of the relative bending of the vertically- and horizontally-bended inlet on the inlet's microwave scattering characteristics. Huang and Liu (2008) have studied the microwave scattering characteristics of the aircraft's surface cracks by RCS measuring. He et al. (2010) have studied the influence of inhomogeneous plasma on the attenuation of planar electromagnetic waves. Sun and Zhang (2008) have summarized the characteristics of F/A-22 and F-35 stealth fighter jets and have proposed a conceptual solution to reduce the antenna aperture size along with the antenna aperture characteristic signal, adopting the technology of low intercept probability. Lu and Wang (2009) have studied the infrared radiation model of the aircraft's surface, analyzed the aircraft's infrared characteristics, and summarized the infrared stealth reduction strategies. Hu and Yu (2011) have studied the applications of multidisciplinary optimization to aircraft's conceptual design, where the aircraft's aerodynamic performance, stealth performance, structure design, general layout and weight constraints are all taken into consideration.

Generally speaking, a series of issues about the stealth aircraft have been studied deeply; however, there are few research reports about the influence of angle changes of the variablesweep wing aircraft on its stealth characteristics. The qualitative and quantitative relationship between the wing's leading edge sweep angle of the variable-sweep wing aircraft and the aircraft's stealth performance still remains unclear. This paper focuses on the stealth characteristics of the variable-sweep wing aircraft. Physical Optics (PO) method and the Equivalent Currents Method (ECM) are used. A numerical simulation is conducted to obtain an analytical report of the RCS characteristics, so as to provide reference for the designing of stealth aircraft.

\section{THEORETICAL BASIS}

The theoretical basis of this study consists of two parts: RCS prediction methods and radar detection probability model.

\section{RCS PREDICTION METHODS}

A series of numerical simulations of RCS characteristics of variable-sweep wing aircraft are conducted in this paper. Scattering surface elements are calculated using PO method. Edge diffraction is calculated by ECM. In PO method, it is assumed that the surface current on the incident point of the wave is equal to the surface current when the incident wave is just on the tangency plane of the incident point.

The formula of PO method is as follows (Yue et al., 2014b):

$$
\begin{aligned}
& \sqrt{\sigma_{p o}}=\left\{\begin{array}{l}
\frac{\hat{\boldsymbol{n}} \cdot\left(\hat{\boldsymbol{e}}_{r} \times \hat{\boldsymbol{h}}_{i}\right) e^{j \frac{2 \pi}{\lambda} \boldsymbol{r}_{o} \cdot(\hat{s}-\hat{\boldsymbol{i}})}}{\sqrt{\pi}|\boldsymbol{p}|^{2}} \sum_{m=1}^{3} \xi_{m} \quad \boldsymbol{p} \neq 0 \\
\frac{j 2 \sqrt{\pi}}{\lambda} \hat{\boldsymbol{n}}^{2} \cdot\left(\hat{\boldsymbol{e}}_{r} \times \hat{\boldsymbol{h}}_{i}\right) e^{j \frac{2 \pi}{\lambda} r_{o} \cdot(\hat{\boldsymbol{s}}-\hat{\boldsymbol{i}})} A \quad \boldsymbol{p}=0
\end{array}\right. \\
& \xi_{m}=\boldsymbol{p} \cdot \boldsymbol{L}_{m} e^{j k r_{m} \cdot(\hat{\boldsymbol{s}}-\hat{\boldsymbol{i}})} \frac{\sin \left(\frac{\pi}{\lambda} \boldsymbol{L}_{m} \cdot(\hat{\boldsymbol{s}}-\hat{\boldsymbol{i}})\right)}{\frac{\pi}{\lambda} \boldsymbol{L}_{m} \cdot(\hat{\boldsymbol{s}}-\hat{\boldsymbol{i}})}
\end{aligned}
$$

where:

$\sqrt{\sigma_{p o}}$ : RCS of a single surface element, in $\mathrm{m}^{2} ; \hat{\boldsymbol{n}}$ : outward normal unit vector of the surface element; $\hat{\boldsymbol{e}}_{\boldsymbol{r}}$ : direction of electric field on the receiving antenna; $\hat{\boldsymbol{h}}_{i}$ : direction of the incident wave's magnetic field; ". " is the dot product and " $x$ " is the cross product; $j$ : imaginary unit, and $j^{2}=1 ; \lambda$ : incident wavelength, in $\mathrm{m}$; $\boldsymbol{r}_{0}$ : reference point on the surface element; $\hat{\boldsymbol{s}}$ : scattering wave's direction; $\boldsymbol{i}$ : incident wave's direction; $\boldsymbol{p}=\hat{\boldsymbol{n}} \times(\hat{\boldsymbol{s}}-\hat{\boldsymbol{i}}) ; A$ : area of the surface element, in $\mathrm{m}^{2} ; L_{\mathrm{m}}$ : magnitude and direction of the $m^{\text {th }}$ edge; $k$ : wave number, $2 \pi / \lambda ; \boldsymbol{r}_{\mathrm{m}}$ : vector from the reference point $\boldsymbol{r}_{0}$ to the midpoint of the $m^{\text {th }}$ edge.

The formula of ECM is as follows:

$$
\begin{aligned}
& \sqrt{\sigma_{e c m}}=\frac{1}{\sqrt{\pi} \sin \theta}\left[\left(\boldsymbol{E}_{0}^{i} \cdot \boldsymbol{t}\right) f_{\boldsymbol{s}} \times(\boldsymbol{s} \times \boldsymbol{t})\right. \\
& \left.-Z_{0}\left(\boldsymbol{H}_{0}^{i} \cdot \boldsymbol{t}\right) g \boldsymbol{s} \times \boldsymbol{t}\right] \cdot e^{-j 2 k k_{t} \cdot s} \frac{\sin (\mathrm{kl} \cdot \boldsymbol{s})}{\mathrm{k} \cdot \boldsymbol{s}}
\end{aligned}
$$

where:

$t$ : mandatory edge unit vector's direction; $i$ incident wave; $E_{0}{ }^{i}$ strength of the incident electric field; $f$ and $g$ : Yoffie Rousseff 
diffraction coefficients; $s$ : scattering wave's direction; $Z_{0}$ : wave impedance in vacuum; $H_{0}{ }^{i}$ : strength of the incident magnetic field; $r_{t}$ : position vector of the middle of the edge; $l$ : vector of the edge; $\theta$ : angle between $i$ and $t$. Other symbols' definition can be found in Yue et al. (2014a, 2014c, 2014d, 2015).

The superposition formula of the RCS of variable-sweep wing aircraft is as follows:

$$
\sigma=\left|\sum_{i=1}^{n}\left(\sqrt{\sigma_{p o}}\right)_{i}+\sum_{i=1}^{n}\left(\sqrt{\sigma_{e c m}}\right)_{i}\right|^{2}
$$

The arithmetic average RCS of the aircraft is shown as follows:

$$
\bar{\sigma}_{n \sim N}^{\beta}=\frac{1}{N-n+1} \sum_{\varphi=n}^{N} \sigma_{\varphi}^{\beta}
$$

The unit-conversion formula of the aircraft's RCS is shown as follows:

$$
\sigma_{\mathrm{dBm}}^{2}=101 \mathrm{~g} \sigma
$$

where:

$\sigma$ : RCS of the variable-sweep wing aircraft, in $\mathrm{m}^{2} ; \sigma_{\varphi}^{\beta}$ : RCS in $\mathrm{m}^{2}$ when the pitching angle of the incident wave is $\beta$ and the azimuth angle of the aircraft is $\varphi ; \bar{\sigma}_{n \sim N}^{\beta}$ : average value of RCS when the pitching angle of the incident wave is $\beta$ and the azimuth angle $\varphi$ ranges from $n$ to $N ; \sigma_{\mathrm{dBm}^{2}}$ : RCS of the variablesweep wing aircraft, in $\mathrm{dBm}^{2}$.

\section{RADAR DETECTION PROBABILITY MODEL}

The probability that the aircraft is detected by a pulse Doppler radar can be estimated as follows Yue et al. (2010):

$$
P_{D k}=\exp \left(-\frac{9.5 \sqrt{\Omega}(4 \pi)^{3} k_{b} T_{0} B_{n} F_{n} L R^{4}}{\sqrt{\Delta \theta_{\alpha} f_{r}} P_{t} G^{2} \lambda^{2} E \sigma_{\varphi \gamma \beta}}\right)
$$

where:

$P_{D k}$ : detection probability of the target; $\Delta \theta_{\alpha}$ : horizontal width of the radar lobe, in degrees; $f_{\mathrm{r}}$ : radar's pulse repetition frequency $(\mathrm{PRF})$, in $\mathrm{Hz}$; $\Omega$ : radar's scanning angular velocity, in degrees/s; $P_{t}$ : radar transmitting power, in Watts $(\mathrm{W})$; $G$ : antenna gain, in $\mathrm{dB} ; E$ : pulse compression ratio of the pulse compression radar; $k_{b}$ : Boltzmann constant, and its value is $1.38 \times 10^{-23} \mathrm{Ws} / \mathrm{K} ; T_{0}$ : standard room temperature, and its value is $290 \mathrm{~K}$; $B_{n}$ : noise bandwidth in the receiver, in $\mathrm{Hz} ; F_{n}$ : noise coefficient in the receiver, in $\mathrm{dB}$; $L$ : total system losses, in $\mathrm{dB}$; $R$ : detection distance, in $\mathrm{m} ; \sigma_{\varphi \gamma \beta}:$ RCS of the aircraft when the azimuth angle is $\varphi$, the rolling angle is $\gamma$, and the pitching angle is $\beta$, in $\mathrm{m}^{2}$.

In order to demonstrate the influence of the varying sweep angle on the detection probability, we choose a typical groundto-air searching situation for simplicity. In this situation, the aircraft is assumed to fly right towards the searching radar, and only the pitching angle varies with the detection distance. The pitching angle of the incident wave relative to the aircraft can be calculated as follows:

$$
\begin{aligned}
& \beta=\beta_{f}-\frac{180}{\pi} \arcsin \left(\frac{h_{d}-h_{m}}{R}\right) \\
& l \leq\left\{\sqrt{\left(h_{m}+R_{d}\right)^{2}-R_{d}^{2}}+\sqrt{\left(h_{d}+R_{d}\right)^{2}-R_{d}^{2}}\right\}
\end{aligned}
$$

where:

$\beta$ : pitching angle of the incident wave relative to the aircraft, in degrees. $\beta$ is negative when the incident wave is below the aircraft; $\beta_{f}$ pitching angle of the aircraft relative to the ground, in degrees. It is negative when the aircraft's nose is upward; $h_{d}$ : vertical distance between the aircraft and the sea level, in $\mathrm{km} ; h_{m}$ : vertical distance between the radar and the sea level, in $\mathrm{km} ; R_{d}$ : radius of the Earth and it is $6,371 \mathrm{~km}$ in general.

\section{ALGORITHM VERIFICATION IN MICROWAVE ANECHOIC CHAMBER}

In order to verify the effectiveness of the numerical simulation method that combines the PO method and the ECM, we have conducted a verification experiment in a microwave anechoic chamber.

The experiment procedure is as follows:

- Use CATIA to build a 3-D digital model of the aircraft, as shown in Fig. 1a.

- Use a 3-D printer to make a 1:36 scaled wax model, as shown in Fig. 1 b.

- Transform the wax model into a sand model cavity and caste aluminum in the sand mold cavity to get a 3-D model in the state of cast-aluminum model, as shown in Fig. 1c.

- Conduct the RCS measurement experiment on the cast-aluminum model in the microwave anechoic chamber, as shown in Fig. 1d. 
The initial conditions of numerical simulation are: the incident wave is at $\mathrm{X}$ band and of horizontal polarization. The pitching angle of the incident wave is $0^{\circ}$. The azimuth angle of the aircraft is between $0^{\circ} \sim 180^{\circ}$. Use PO method and ECM to do the numerical simulation on the 3 -D digital prototype shown in Fig. 1a, and the RCS characteristics curve can be obtained as shown in Fig. 2.

According to the similarity principle, the incident wavelength should be $0.83 \mathrm{~mm}$ when conducting the RCS measurement experiment in the microwave anechoic chamber. And the incident wave should also be of horizontal polarization. The measurement results are shown in Fig. 2.

From the comparison between the numerical simulation and experimental results, we can see that the two RCS characteristic curves basically match each other. After verifying the scientific character and accuracy of the numerical simulation method, we can use this method to analyze the RCS characteristics of the aircraft.

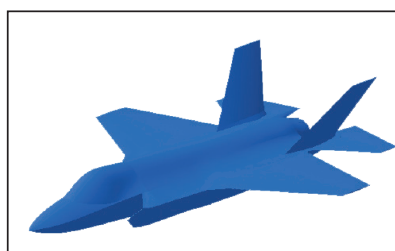

(a)

(c)

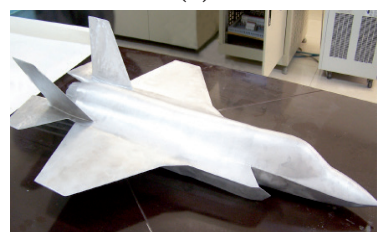



(b)

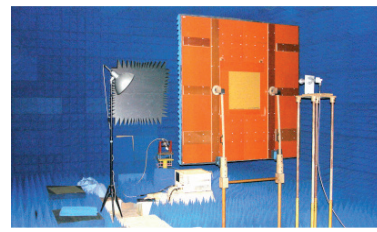

(d)
Figure 1. Microwave chamber experiment. (a) 3-D digital model; (b) Wax model; (c) Cast-aluminum model; (d) Microwave chamber.

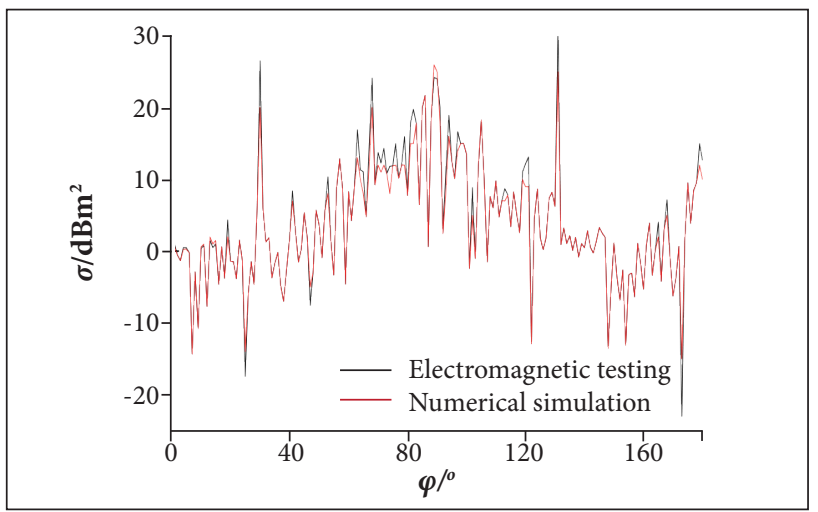

Figure 2. Comparison of the aircraft's RCS between numerical simulation and experiment results.

\section{NUMERICAL SIMULATION OF AIRCRAFT'S RCS}

Numerical simulation of aircraft's RCS consists of four components: CATIA modeling of the variable-sweep wing aircraft, numerical simulation of the aircraft's RCS, analysis of the impact of wing leading edge's sweep angle on aircraft's RCS, and the probability of being detected by a searching radar.

\section{CATIA MODELING OF THE VARIABLE-SWEEP WING AIRCRAFT}

The variable-sweep wing aircraft scheme in this paper has adopted features as follows: single-seat, twin-engine, twin vertical tails extraversion, variable-sweep wing aerodynamic layout, the band edge of the wing and blended wing body. Double S-shaped bend inlets are adopted on both sides of the fuselage, and three bomb bays are buried into the fuselage. Figure 3 shows the layout of the aircraft.

The CATIA model of the variable-sweep wing aircraft uses parametric designing method to design the wing leading edge's sweep angle, which is defined as $\chi$ (Fig. 4). Other basic parameters of the aircraft remain unchanged, in which the length of the aircraft is $22 \mathrm{~m}$.

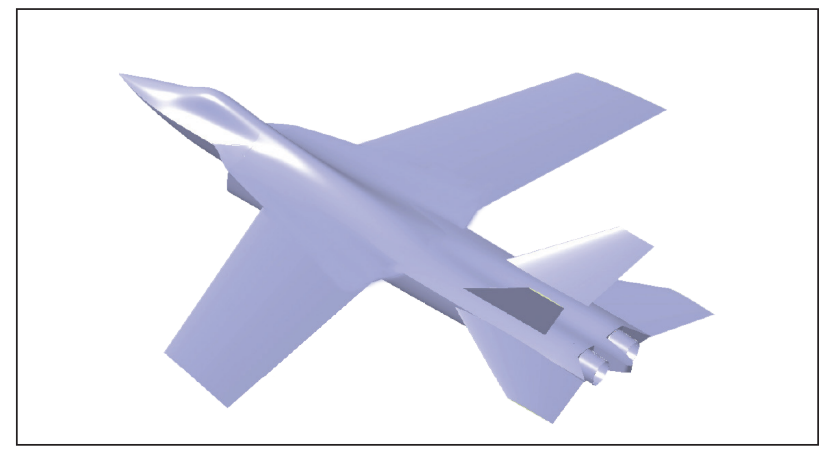

Figure 3. CATIA model of variable-sweep wing aircraft.

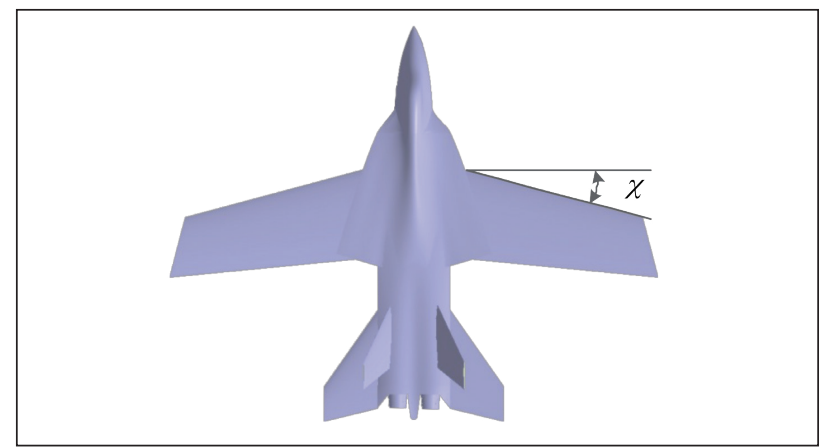

Figure 4. Schematic diagram of wing leading edge's sweep angle. 


\section{NUMERICAL SIMULATION OF THE AIRCRAFT'S RCS}

CATIA is used in this study to establish a 3-D digital model for the variable-sweep wing aircraft. Analysis manager module is used to generate the 3-D grids (Fig. 5). Based on PO method and ECM, we import the aircraft 3-D grids to the RCS calculating program written in Matlab language. Under the conditions that the radar incident wave is at X band and of horizontal polarization, the pitching angle of the incident wave is $-5^{\circ}, 0^{\circ}$ and $+5^{\circ}$, and the azimuth angle of the aircraft is between $0^{\circ} \sim 360^{\circ}$. In this section, we conduct a numerical simulation of the RCS of the variable-sweep wing aircraft and then obtain the RCS characteristic curve of the variable-sweep wing aircraft when $\chi=15^{\circ}$ (Fig. 6).

In the polar coordinates of the aircraft's RCS characteristic, the azimuth angle $0^{\circ}$ corresponds to the head direction of the aircraft and the azimuth angle $180^{\circ}$ corresponds to the tail direction of the aircraft. And when the azimuth angle is $90^{\circ}$, it corresponds to the right side of the aircraft.

From the data shown in Fig. 6, we can obtain some conclusions here: when $\chi=15^{\circ}$ and the pitching angle of the incident wave is $0^{\circ}$, the arithmetic average value of the aircraft's RCS between

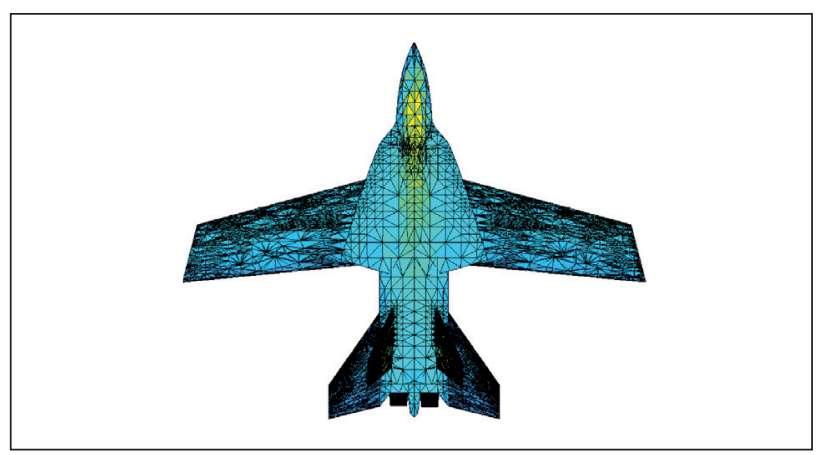

Figure 5. 3- $D$ grids of aircraft when $\chi=15^{\circ}$.

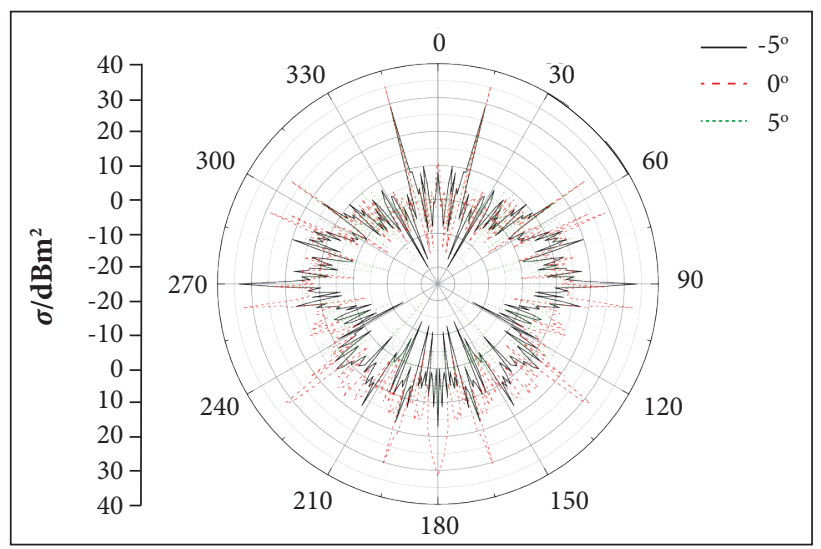

Figure 6. RCS characteristics of the aircraft when $\chi=15^{\circ}$. $\pm 10^{\circ}$ is $3.59 \mathrm{dBm}^{2}$, the arithmetic average value of the aircraft's RCS between $\pm 30^{\circ}$ is $20.43 \mathrm{dBm}^{2}$, and the RCS peak value in the head direction of the aircraft appears symmetrically and its value is $35.29 \mathrm{dBm}^{2}$, appearing at the azimuth angles of $+15^{\circ}$ and $-15^{\circ}$.

When the pitching angle of the incident wave is $-5^{\circ}$, the arithmetic average value of the aircraft's RCS between $\pm 10^{\circ}$ is $4.50 \mathrm{dBm}^{2}$, the arithmetic average value of the aircraft's RCS between $\pm 30^{\circ}$ is $14.51 \mathrm{dBm}^{2}$, and the RCS peak value in the head direction of the aircraft appears symmetrically and its value is $29.07 \mathrm{dBm}^{2}$, appearing at the azimuth angles of $+15^{\circ}$ and $-15^{\circ}$.

When the pitching angle of the incident wave is $+5^{\circ}$, the arithmetic average value of the aircraft's RCS between $\pm 10^{\circ}$ is $1.58 \mathrm{dBm}^{2}$, the arithmetic average value of the aircraft's RCS between $\pm 30^{\circ}$ is $13.89 \mathrm{dBm}^{2}$, and the RCS peak value in the head direction of the aircraft appears symmetrically and its value is $28.56 \mathrm{dBm}^{2}$, appearing at the azimuth angles of $+15^{\circ}$ and $-15^{\circ}$.

We can thus draw a qualitative conclusion that the azimuth angle of the RCS peak value of the variable-sweep wing aircraft is equal to the sweep angle $\chi$.

\section{ANALYSIS OF THE IMPACT OF THE AIRCRAFT'S SWEEP ANGLE ON ITS RCS CHARACTERISTICS}

This section is an in-depth study of the qualitative conclusions obtained from the previous section. A series of numerical simulations of the RCS of the variable-sweep wing aircraft is conducted and the equivalence between the aircraft RCS peak azimuth angle and $\chi$ is then analyzed.

The purpose of calculating the average value of the aircraft's RCS is to analyze the influence of the variation of the sweep angle on the aircraft's RCS characteristics, from the perspective of mathematical statistics and the perspective of aircraft conceptual design and stealth design.

The leading edge's sweep angle $\chi$ varies from $0^{\circ}$ to $66^{\circ}$, at an interval of $3^{\circ}$, and consequently 23 kinds of 3 -D digital prototypes of the variable-sweep wing aircraft can be obtained. When calculating each aircraft prototype's RCS, the grids covering the whole aircraft are firstly generated, the radar incident wave is set to be at $\mathrm{X}$ band and of the horizontal polarization, and the pitching angle is $0^{\circ} ; 23$ different characteristic curves of the aircraft's RCS can then be obtained. In this series of numerical simulations, the variable-sweep wing aircraft's grids are shown in Fig. 7, where $\chi$ is equal to $39^{\circ}, 54^{\circ}$ and $66^{\circ}$, respectively, and the RCS characteristic curves for these three different variablesweep wing aircraft are shown in Fig. 8. 


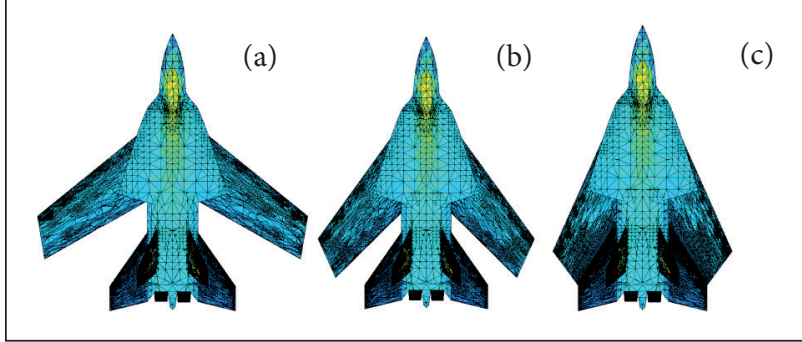

Figure 7. Trellis diagram in the type for $\chi$ of aircraft. (a) $\chi=39^{\circ}$; (b) $\chi=54^{\circ}$; (c) $\chi=66^{\circ}$.

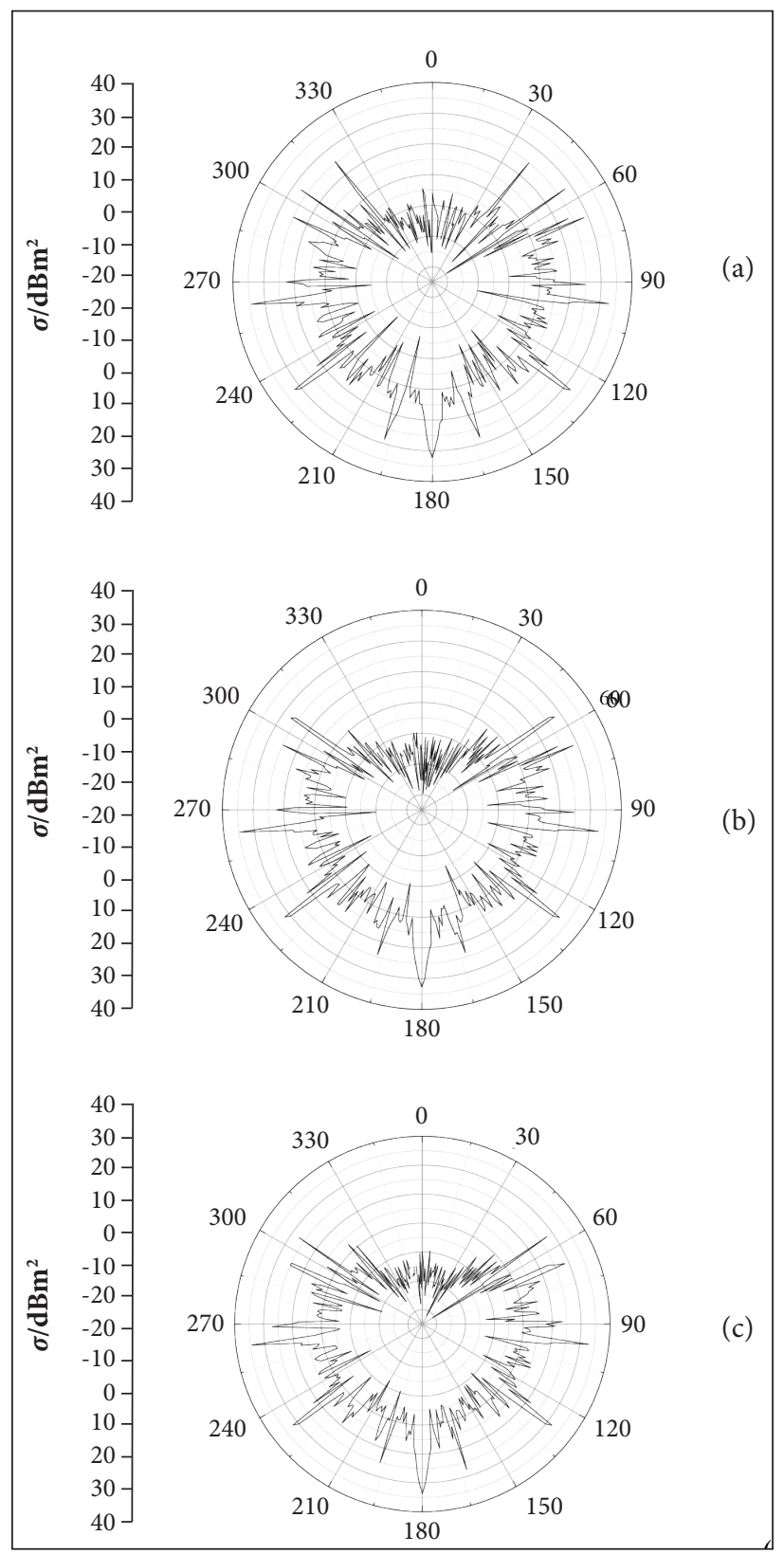

Figure 8. RCS characteristics in the type for $\chi$ of aircraft. (a) $\chi=39^{\circ}$; (b) $\chi=54^{\circ} ; \chi=66^{\circ}$.
From the data shown in Figs. 8a to 8c, we can obtain some conclusions here: (1) when the wing leading edge's sweep angle is $39^{\circ}$, the RCS arithmetic average value between $\pm 10^{\circ}$ of the aircraft is $-1.02 \mathrm{dBm}^{2}$, and the RCS arithmetic average value between $\pm 30^{\circ}$ of the aircraft is $-1.61 \mathrm{dBm}^{2}$. The RCS peak value, appearing symmetrically in the aircraft's head direction, is $24.85 \mathrm{dBm}^{2}$ and appears at both $+39^{\circ}$ and $-39^{\circ}$ azimuth angles. This proves that, in the head direction of the aircraft, the azimuth angle corresponding to the RCS peak value is equal to the wing leading edge's sweep angle; (2) when the wing leading edge's sweep angle is $54^{\circ}$, the RCS arithmetic average value between $\pm 10^{\circ}$ of the aircraft is $-5.901 \mathrm{dBm}^{2}$, and the RCS arithmetic average value between $\pm 30^{\circ}$ of the aircraft is $-5.59 \mathrm{dBm}^{2}$. The RCS peak, appearing symmetrically in the head direction of the aircraft, is $26.21 \mathrm{dBm}^{2}$ and appears at both $+54^{\circ}$ and $-54^{\circ}$ azimuth angles. This proves that, in the head direction of the aircraft, the azimuth angle corresponding to the RCS peak value is equal to the wing leading edge's sweep angle; (3) when the wing leading edge's sweep angle is $66^{\circ}$, the RCS arithmetic average value between $\pm 10^{\circ}$ of the aircraft is $-4.68 \mathrm{dBm}^{2}$, and the RCS arithmetic average value between $\pm 30^{\circ}$ of the aircraft is $-6.198 \mathrm{dBm}^{2}$. The RCS peak value appears twice symmetrically in the head direction of the aircraft, and the peak values are 27.72 and $25.1 \mathrm{dBm}^{2}$, appearing at both $\pm 55^{\circ}$ and $\pm 66^{\circ}$ azimuth angles. This proves that, in the head direction of the aircraft, the azimuth angle corresponding to the RCS peak value is equal to the wing leading edge's sweep angle. For another RCS peak, the aircraft forward azimuth is equal to the horizontal tail leading edge sweep angle, which is $\pm 55^{\circ}$.

Based on the mathematical statistics and comparative analysis of all the 23 aircraft RCS characteristic curves obtained from the simulations, the relationship curve between the RCS peak value and the sweep angle $\chi$ is shown in Fig. 9, and the relationship curve between the corresponding azimuth angle of the aircraft RCS forward peak value and the sweep angle $\chi$ is shown in Fig. 10, the relationship curves between the aircraft RCS forward arithmetic average value and sweep angle $\chi$ are shown in Fig. 11.

It can be seen from Fig. 9 that, when the wing leading edge's sweep angle $\chi$ is $0^{\circ}$, its aircraft's RCS forward peak value is $36.08 \mathrm{dBm}^{2}$. When $\chi=18^{\circ}$, its aircraft's RCS peak value is $28.19 \mathrm{dBm}^{2}$, and, after unit conversion, we know that the aircraft's RCS peak value when $\chi=18^{\circ}$ is $16.25 \%$ of that when $\chi=0^{\circ}$. When $\chi=33^{\circ}$, its aircraft's RCS peak value is $26.19 \mathrm{dBm}^{2}$, and the converting results show that the aircraft RCS peak value when $\chi=33^{\circ}$ is $10.25 \%$ of that when $\chi=0^{\circ}$. When $\chi=42^{\circ}$, its aircraft's RCS peak value is $29.85 \mathrm{dBm}^{2}$, and 
the converting results show that, when $\chi=42^{\circ}$, the aircraft's RCS peak value is $23.82 \%$ of that when $\chi=0^{\circ}$. When $\chi=54^{\circ}$, its aircraft's RCS peak value is $26.21 \mathrm{dBm}^{2}$, and the converting results show that the aircraft's RCS peak value for $\chi=54^{\circ}$ is $10.30 \%$ of that when $\chi=0^{\circ}$. When $\chi=66^{\circ}$, its aircraft's RCS peak value is $25.10 \mathrm{dBm}^{2}$, and the converting results show that the aircraft's RCS peak value when $\chi=66^{\circ}$ is $7.97 \%$ of that when $\chi=0^{\circ}$. It can be seen that, when the sweep angle of the aircraft increases, the peak value of the RCS decreases non-linearly.

Figure 10 shows that the azimuth angle corresponding to one of the RCS peak value is equal to the aircraft's sweep angle.

From Fig. 11, we can see that:

- The arithmetic average value of the aircraft's RCS between $\pm 10^{\circ}$ is $\bar{\sigma}_{t}^{ \pm 10^{\circ}}$ and, when $\chi=0^{\circ}, \bar{\sigma}_{t}^{ \pm 10^{\circ}}=25.67 \mathrm{dBm}^{2}$; when $\chi=33^{\circ}, \bar{\sigma}_{t}^{ \pm 10^{\circ}}=0 \mathrm{dBm}^{2}$ and, after unit conversion, we know that when $\chi=33^{\circ}, \bar{\sigma}_{t}^{ \pm 10^{\circ}}$ is $0.271 \%$ of that when $\chi=0^{\circ}$. When $\chi=42^{\circ}, \bar{\sigma}_{t}^{ \pm 10^{\circ}}=-4.97 \mathrm{dBm}^{2}$, and when $\chi=42^{\circ}$, $\bar{\sigma}_{t}^{ \pm 10^{\circ}}$ is $0.087 \%$ of that when $\chi=0^{\circ}$.

- The arithmetic average value of the aircraft's RCS between $\pm 30^{\circ}$ is $\bar{\sigma}_{t}^{ \pm 30^{\circ}}$ and, when $\chi=0^{\circ}, \bar{\sigma}_{t}^{ \pm 30^{\circ}}=21.21 \mathrm{dBm}^{2}$; when $\chi=33^{\circ}, \bar{\sigma}_{t}^{ \pm 30^{\circ}}=-0.7 \mathrm{dBm}^{2}$ and, after unit conversion, we know that, when $\chi=33^{\circ}, \bar{\sigma}_{t}^{ \pm 30^{\circ}}$ is $0.644 \%$ of that when $\chi=0^{\circ}$; when $\chi=42^{\circ}, \bar{\sigma}_{t}^{ \pm 30^{\circ}}=-2.67 \mathrm{dBm}^{2}$ and, when $\chi=42^{\circ}$, $\bar{\sigma}_{t}^{ \pm 30^{\circ}}$ is $0.409 \%$ of that when $\chi=0^{\circ}$.

- The arithmetic average value of the aircraft's RCS between $\pm 45^{\circ}$ is $\bar{\sigma}_{t}^{ \pm 45^{\circ}}$ and, when $\chi=0^{\circ}, \bar{\sigma}_{t}^{ \pm 45^{\circ}}=19.53 \mathrm{dBm}^{2}$; when $\chi=33^{\circ}, \bar{\sigma}_{t}^{ \pm 45^{\circ}}=10.07 \mathrm{dBm}^{2}$ and, when $\chi=33^{\circ}$, $\bar{\sigma}_{t}^{ \pm 45^{\circ}}$ is $11.324 \%$ of that when $\chi=0^{\circ}$. When $\chi=42^{\circ}$, $\bar{\sigma}_{t}^{ \pm 45^{\circ}}=13.39 \mathrm{dBm}^{2}$ and, when $\chi=42^{\circ}, \bar{\sigma}_{t}^{ \pm 45^{\circ}}$ is $24.32 \%$ of that when $\chi=0^{\circ}$.

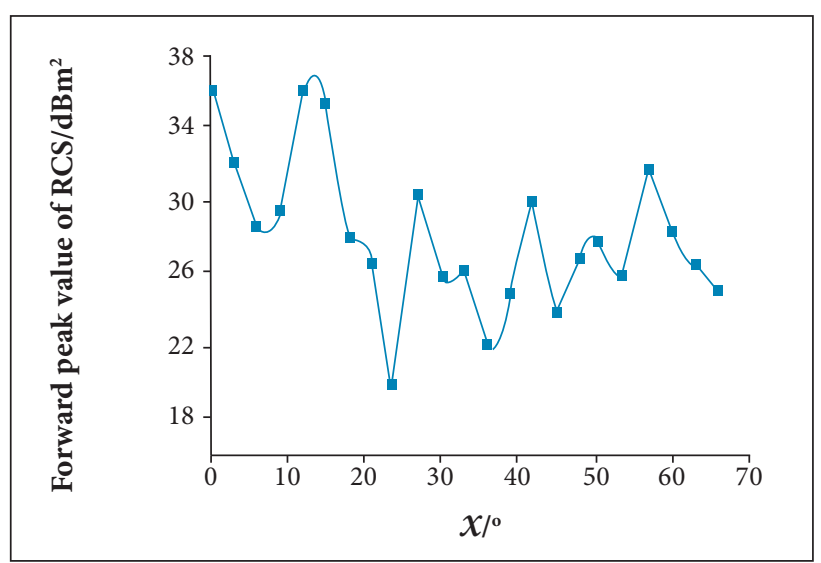

Figure 9. Relation between aircraft's RCS forward peak value and $\chi$.

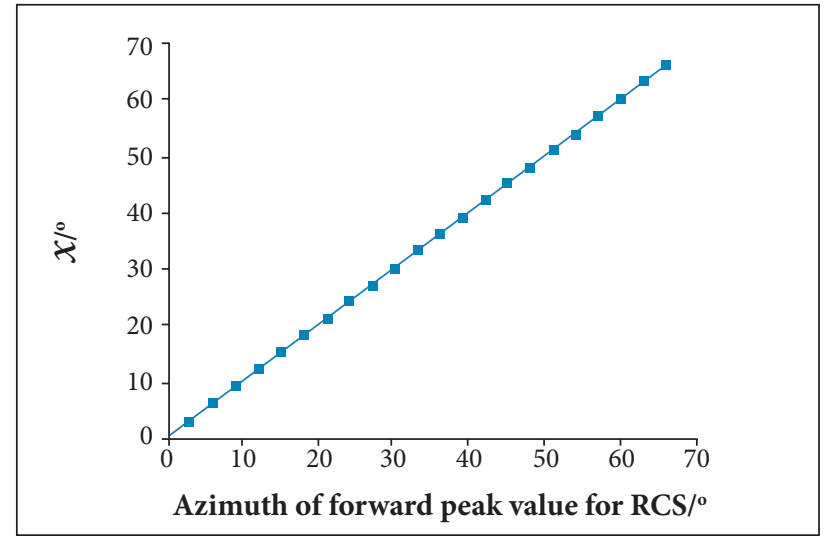

Figure 10. Relation between the corresponding azimuth angle of aircraft's RCS forward peak value and $\chi$.

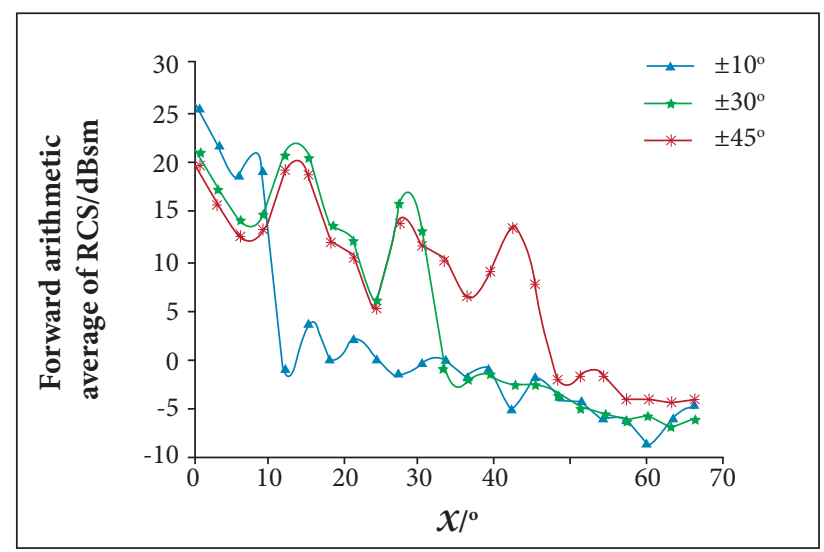

Figure 11. Relation between aircraft's RCS forward arithmetic average value and $\chi$.

\section{CALCULATING THE RADAR DETECTION PROBABILITY}

The purpose of studying the RCS characteristics of the variable-sweep wing aircraft is to reduce the detection probability by the hostile radar. In this section, we study the relationship between the detection probability and the characteristics of the target's RCS and the detection distance.

In a typical ground-to-air searching situation, as mentioned in section Radar Detection Probability Model, the rolling and azimuth angles of the incident wave relative to the aircraft are both $0^{\circ}$, and $\beta_{\rho} h_{\mathrm{d}}, h_{\mathrm{m}}$ of Eqs. 8 and 9 are set to be $-2.5^{\circ}, 8 \mathrm{~km}$, and $3 \mathrm{~km}$, respectively. Figure 12 shows the relationship between the pitching angle of the incident wave and the detection distance.

As shown in Fig. 12, $\beta$ decreases from $-5^{\circ}$ to below $-30^{\circ}$ when the detection distance decreases from 200 to $0 \mathrm{~km}$. The corresponding RCS values of the aircraft under these attitudes can then be calculated using the PO method and 
ECM. The incident wave is at $\mathrm{X}$ band and of horizontal polarization. Figure 13 shows the varying RCS value of the aircraft when it is flying towards the searching radar from $200 \mathrm{~km}$ away. Three types of lines indicate three different sweep angles, which are $\chi=15^{\circ}, 39^{\circ}$ and $54^{\circ}$, respectively.

Figure 13 shows that, when the aircraft is flying towards the radar from $200 \mathrm{~km}$ away, its RCS value varies between about -20 and $10 \mathrm{dBm}^{2}$.

According to the RCS values shown in Fig. 13, the detection probability can be calculated using Eq. 7. The initial parameters of the pulse Doppler radar are set as follows: $\Delta \theta_{\alpha}=7^{\circ}, f_{\mathrm{r}}=300 \mathrm{~Hz}, \Omega=30^{\circ} / \mathrm{s}, P_{\mathrm{t}}=10 \mathrm{MW}, G=36 \mathrm{~dB}$, $\lambda=0.03 \mathrm{~m}, E=900, B_{\mathrm{n}}=0.077 \mathrm{MHz}, F_{\mathrm{n}}=55 \mathrm{~dB}, L=20 \mathrm{~dB}$. RCS values of Fig. 13 are expressed in $\mathrm{dBm}^{2}$, when applied to Eq. 7, they should be converted to $\mathrm{m}^{2}$, with the help of Eq. 6. Figure 14 shows the calculation results of the aircraft's detection probability.

As shown in Fig. 14, when the aircraft is flying right towards the radar from $200 \mathrm{~km}$ away, the detection probabilities are very low when $R$ is beyond about $120 \mathrm{~km}$, for all 3

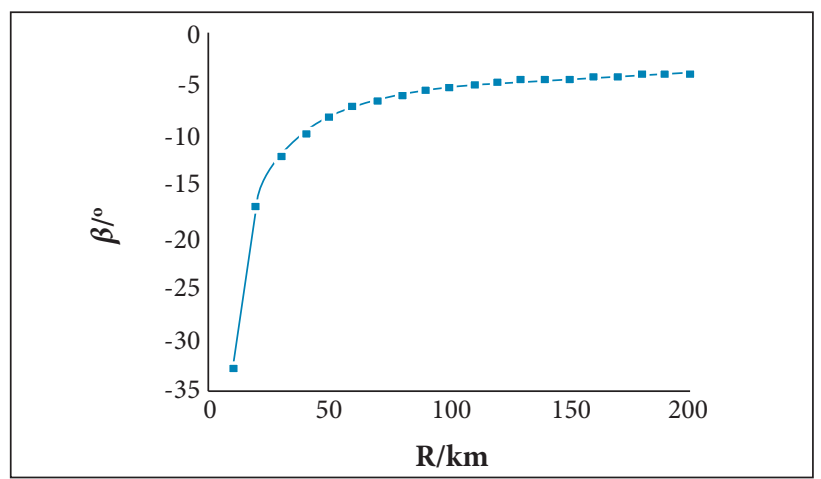

Figure 12. The pitching angle of the incident wave against the detection distance.

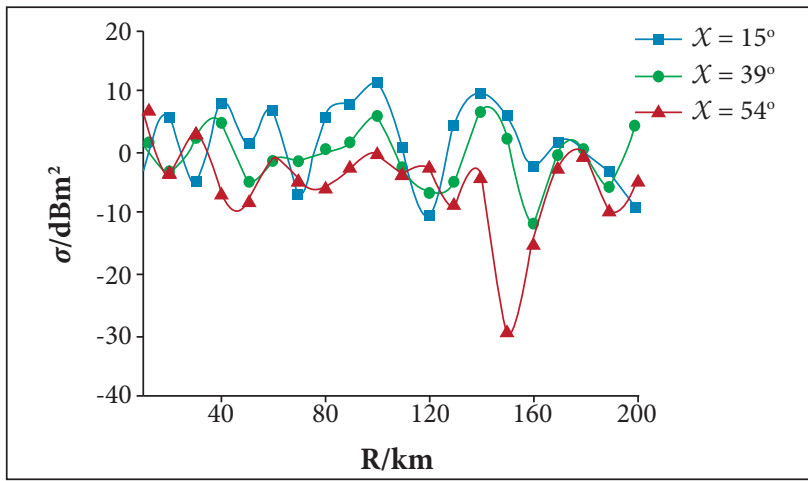

Figure 13. The RCS of variable-sweep wing aircraft against detection distance. types of variable-sweep wing aircrafts. When the distance decreases to within $100 \mathrm{~km}$, the aircraft with a sweep angle of $\chi=54^{\circ}$ performs best and the aircraft with a sweep angle of $\chi=15^{\circ}$ performs worst. When the distance decreases to within $40 \mathrm{~km}$, all 3 types of variable-sweep wing aircrafts perform almost the same.

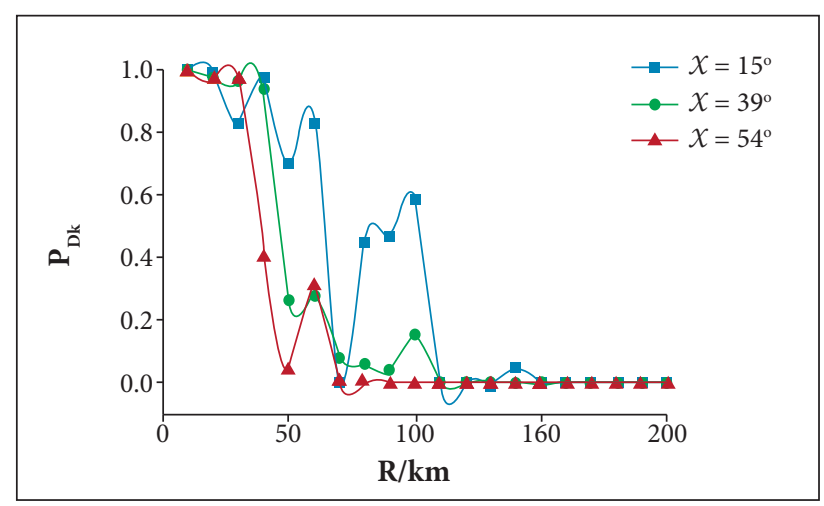

Figure 14. Detection probability of the aircraft when it is flying from $200 \mathrm{~km}$ away.

\section{CONCLUSION}

This paper utilizes the physical optics and the equivalent currents methods to study the RCS characteristics of the variablesweep wing aircraft. Based on the numerical simulations of the aircraft, the following conclusions can be obtained:

The aircraft's RCS forward peak value decreases non-linearly with the sweep angle $\chi$.

The sweep angle of the variable-sweep wing aircraft and the azimuth angle corresponding to one of the RCS peak values are identical.

When $\chi=33^{\circ}, \bar{\sigma}_{t}^{ \pm 30^{\circ}}=-0.7 \mathrm{dBm}^{2}$. After unit conversion, $\bar{\sigma}_{t}^{ \pm 30^{\circ}}$ of the aircraft when $\chi=33^{\circ}$ is $0.644 \%$ of that when $\chi=0^{\circ}$.

The larger the sweep angle of the variable-sweep wing aircraft is, the lower the aircraft's detection probability will be.

We hope that the conclusions of this paper provide some reference and technical support for stealth aircraft's demonstration and designing.

\section{ACKNOWLEDGEMENT}

This work was supported by the Natural Science Foundation of China (51375490). 


\section{REFERENCES}

Bai, Z.D. and Liu, H., 2007, "Parametric Modeling and Optimization of Observability Fuselage in Aircraft Conceptual Design", Journal of Beijing University of Aeronautics and Astronautics, Vol. 33, No. 12, pp. 1391-1394.

Bao, J.B. and Wang, G.L., 2012, "Optimization and Experimental Verification for Aerodynamic Scheme of Flying-Wing", Journal of Beijing University of Aeronautics and Astronautics, Vol. 38, No. 2, pp. 180-184.

He, X., Chen, J.P. and Ni, X.W., 2010, "Attenuation of Planar Electromagnetic Waves by Inhomogeneous Plasma", High Power Laser and Particle Beams, Vol. 22, No. 9, pp. 2115-2118.

Hu, T.Y. and Yu, X.Q., 2011, "Preliminary Design of Unconventional Configuration Aircraft Using Multidisciplinary Design Optimization", Acta Aeronautica et Astronautica Sinica, Vol. 32, No. 1, pp. 117-127.

Huang, P.L. and Liu, Z.H., 2008, "Research on Electromagnetic Scattering Characteristics of Slits on Aircraft", Acta Aeronautica et Astronautica Sinica, Vol. 29, No. 3, pp. 675-680.

Ji, J.Z., Wu, Z. and Liu, Z.H., 2009, "Research on the Curvature Parameter in the S-Shaped Inlet's Stealth Design", Journal of Xidian University, Vol. 36, No. 4, pp. 746-750.

Lu, J.W. and Wang, Qi., 2009, "Aircraft-Skin Infrared Radiation Characteristics Modeling and Analysis", Chinese Journal of Aeronautics, Vol. 22, No. 5, pp. 493-497.

Nangia, R.K. and Palmer, M.E., 2005, "A Comparative Study of UCAV Type Wing Planforms-Aero Performance and Stability Considerations", AIAA-2005-5078.

Sun, C. and Zhang, P., 2008, "LO Requirements and Solutions of Avionics/RF System for Advanced Aircraft", Acta Aeronautica et Astronautica Sinica, Vol. 29, No. 6, pp. 1472-1481.
Tom, R.B. and Alfred, G.S., 2010, "Optimization of Aircraft Configuration for Minimum Drag", AIAA-2010-3000.

Vogel, B.S., 2005, "Boeing X-45 UCAVs Complete Simulated Mission", Janes Defence Industry, UK.

Wood, R.M. and Bauer, S.X.S., 2001, "Flying Wings/Flying Fuselage", AIAA-2001-0311.

Yue, K.Z., Hou, Z.Q. and Yang, Y.K., 2010, "Markov Model of Efficiency of Volleying Anti-Ship Missiles Piercing into Ship-Bone Antiaircraft Missiles", Journal of System Simulation, Vol. 22, No. 6, pp. $1472-1475$.

Yue, K.Z., Liu W.L., Li G.X., Ji J.Z. and Yu D.Z., 2015, "Numerical Simulation of RCS for Carrier Electronic Warfare Airplanes", Chinese Journal of Aeronautics, Vol. 28, No. 2, pp. 545-555.

Yue, K.Z., Gao, Y., Li, G.X. and Yu, D.Z., 2014a, "Conceptual Design and RCS Performance Research of Shipborne Early Warning Aircraft", Journal of Systems Engineering and Electronics, Vol. 25, No. 6, pp. 968-976.

Yue, K.Z., Sun, C. and Ji, J.Z., 2014b, "Numerical Simulation on the Stealth Characteristics of Twin-Vertical-Tails for Fighter", Journal of Beijing University of Aeronautics and Astronautics, Vol. 40, No. 2, pp. 160-165.

Yue, K.Z., Sun, C., Liu, H. and Su, M., 2014c, "Numerical Simulation on the RCS of Combat Aircraft for Mounted Missile", Systems Engineering and Electronics, Vol. 36, No. 1, pp. 62-67.

Yue, K.Z., Tian, Y.F., Liu, H. and Han, W., 2014d, "Conceptual Design and RCS Property Research of Three-Surface Strike Fighter", International Journal of Aeronautical and Space Sciences, Vol. 15, No. 3, pp. 309-319. 